\title{
OSCILLATORY INTERLAYER MAGNETIC COUPLING OF SPUTTERED Fe/Nb SUPERLATTICES*
}

J.E. Mattson, Eric E. Fullerton, C.H. Sowers, Y.Y. Huang, G.P. Felcher, and S.D. Bader Materials Science Division Argonne National Laboratory, Argonne, IL 60439

ANL/CP-76458

DE93 004191

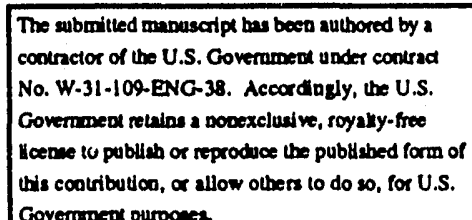

Governineux purposer.

37th Magnetism and Magnetic Materials Conference, Houston, Texas, December 1-4, 1992, Proceedings to be published in Journal of Applied Physics

jmc

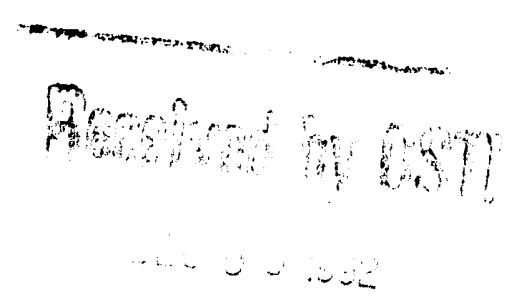

*Research supported in by the U.S. Department of Energy under contract \#W-31-109-ENG-38 and NSF MRG contract No. DMR 86-03304 (JEM). 


\title{
OSCILLATORY INTERLAYER MAGNETIC COUPLING OF SPUTTERED Fe/Nb SUPERLATTICES*
}

\author{
J.E. Mattson, Eric E. Fullerton, C.H. Sowers, Y.Y. Huang, G.P. Felcher \\ and S.D. Bader \\ Material Science Division, Argonne National Laboratory, Argonne, IL 60439
}

The saturation field of sputtered $\mathrm{Fe} / \mathrm{Nb}$ superlattices oscillates as a function of the $\mathrm{Nb}$ thickness with a periodicity of $\sim 9 \AA$. In contrast to the case of $\mathrm{Fe} / \mathrm{Cr}$ superlattices, the concurrent magnetoresistance oscillations were found to be very weak. Yet polarized neutron reflection measurements confirm that the $\mathrm{Fe} / \mathrm{Nb}$ superlattices with high saturation field possess a magnetic ground state of the +-+- type. Neutron and $\mathrm{x}$-ray measurements indicate that, while the crystalline and antiferromagnetic order is well developed along the thickness of the film, the average lateral size of the crystallites (as well as of the magnetic domains) is quite small. This effect (thought to be related to the gross mismatch of the iron and the niobium crystal lattices) may be the cause of the high overall resistance of the material, and its weak dependence on the magnetization.

PACS \#: 72.15.Gd; 75.70Fr; 78.20.Ls; 81.15.Cd 


\section{INTRODUCTION}

A recent set of experiments on $\mathrm{Fe} / \mathrm{Nb}$ sputtered superlattices showed oscillatory magnetic coupling of the Fe layers as a function of the $\mathrm{Nb}$ spacer thickness ${ }^{1}$. Figure 1 presents some of the results obtained by magnetometry and resistivity measurements. Both the remanent magnetization and the magnetoresistance show an oscillatory behavior with the thickness of the niobium layer with a periodicity of $\sim 9 \AA$. Such behavior is similar to that found in an increasingly large class of $3 d / 3 d$ and $3 d / 4 d$ superlattices, of which $\mathrm{Fe} / \mathrm{Cr}$ was the prototype. ${ }^{2}$ What sets apart $\mathrm{Fe} / \mathrm{Nb}$, however, is the fact that the magnetoresistance oscillations have an unusually low amplitude $(\leq 0.3 \%)$ which requires precise measurements to make positive identification. Under these circumstances, we question if, as for $\mathrm{Fe} / \mathrm{Cr}$, the magnetic state for the superlattices with highest magnetoresistance is indeed an inter-layer antiferromagnet, with +-+ - coupling between subsequent Fe layers. To check this, polarized neutron reflectivity measurements were performed on a $[\mathrm{Fe}(15 \AA) / \mathrm{Nb}(15 \AA)]_{100}$ sample which exhibit antiferromagnetic (AF) behavior.

\section{NEUTRON REFLECTION MEASUREMENTS}

The film used for neutron reflection measurements was grown on a 1-in. diameter silicon wafer $1 / 8$-in. thick at ambient temperature by dc magnetron sputtering. The sputtering pressure was 3 mTorr Ar (99.999\% purity) in a chamber of base pressure $\sim 2 \mathrm{x}$ $10^{-9}$ Torr. Sputtering rates were monitored with quartz crystal monitors, and the bilayer thickness was determined by neutron and x-ray diffraction. The neutron measurements were performed at room temperature at the reflectometer POSY I at the Intense Pulsed Neutron Source at Argonne National Laboratory. A residual field of 30 Oe was used so as to maintain the neutron polarization before reaching the sample. The details of the instrument are described in Ref. 3. In this experiment, cold neutrons of all wavelengths were used, and were sent to impinge upon the sample's surface at an angle of grazing incidence $\theta_{i}$ of the order of $2^{\circ}$. Neutrons reflected from the superlattice were measured in a 
small, one-dimensional position sensitive detector, with an azimuthal opening angle of $\Delta \theta_{1}=1.5^{\circ}$

The reflectivity was found to be totally independent of the neutron spin state and the spin averaged quantity is presented in Fig. 2. Only at higher fields, capable of inducing a sizable net magnetization, were the reflectivities for the two spin states different. ${ }^{4}$ For instance at $5 \mathrm{kOe}$, (above the saturation field of the sample) the spin-dependence of the reflectivity is consistent with an induced moment of $0.9 \mu_{\mathrm{B}}$ per Fe atom. This value is in agreement with the reduced moment determined from magnetization measurements. ${ }^{1}$ The most important feature of Fig. 2 is the presence of a Bragg reflection as indicated by the arrow. The Bragg reflection occurs at the scattering vector $q=0.106 \AA^{-1}$, which corresponds to a $d$-spacing of $d=2 \pi / q=59.4 \AA$. This is just twice the superlattice modulation wavelength $\Lambda=29.7 \AA$ of the sample and is expected for a Bragg reflection due to $\mathrm{a}+-+-$ antiferromagnetic ordering of the Fe layers.

Also presented in Fig. 2 is the calculated reflectivity for the film. ${ }^{5}$ The mean density of the film was fitted to $98 \%$ of the bulk values for $\mathrm{Fe}$ and $\mathrm{Nb}$ to provide the best match with the experimental value of the critical momentum transfer $q_{c}$ (shown in Fig. 2). The rapid oscillations visible in the region immediately beyond $q_{c}$ are due to the total film thickness of $2980 \AA$. To fit the width of the AF peak the magnetic coherence length had to be restricted to $\approx 16$ bilayers, or to a thickness of $480 \AA$. The height of the AF peak is calculated to correspond to an iron moment of only $0.29 \mu_{\mathrm{B}}$, which is about a third of the mornent induced in a magnetic field. To understand why such a low value of the moment matches the experimental results, a closer look has to be given to the experimental geometry.

Figure 3 shows a contour plot of the scattered neutron intensities from an incident beam set at $\theta_{i}=1.92^{\circ} .6$ The abscissa gives the angle of the exit beam with respect to the incident beam. The specularly reflected beam is given by $\theta_{\mathrm{f}}=\theta_{\mathrm{i}}=1.92^{\circ}$ regardless of the neutron wavelength $\lambda$. In addition to the specular intensity, the detector records a sharp 
band of intensity, centered around $\mathrm{q}=() .106 \AA^{-1}$, for a wide range of angles of the exit beam. However, the moment of $\left(0.29 \mu_{\mathrm{B}}\right.$ was determined by fitting only the reflectivity in a narrow region around $\theta_{i}+\theta_{f}=3.84^{\circ}$ and not the integrated intensity for $q=0.106 \AA-1$. Therefore, it is not surprising that the AF moment obtained is artificially low. The spread of the scattered magnetic intensity may be due to misalignment of the propagation of the AF structures in different domains, or, more likely, to finite in-plane size of the domains. Unfortunately, the spread of the scattered intensity exceeds the range of angles subtended by the detector, and, thus, an accurate assessment of the lateral size, or angular misorientation of the domains is beyond the capabilities of the present experiment.

\section{X-RAY DIFFRACTION}

In view of the considerable disorder exhibited by the magnetic structure, the crystalline structure of these films was characterized by both low-and high-angle $x$-ray diffraction. Unfortunately, the electron density of $\mathrm{Fe}$ and $\mathrm{Nb}$ are nearly identical, which limits the quantitative information obtainable from the low-angle $x$-ray spectra. ${ }^{1}$ The highangle spectrum of an $[\mathrm{Fe}(20 \AA) / \mathrm{Nb}(18 \AA)] 75$ superlattice is shown in Fig. 4a. Well resolved superlattice peaks are observed, which confirms the superlattice structure of the samples. Both the $\mathrm{Fe}$ and $\mathrm{Nb}$ layers grow oriented with the $\mathrm{bcc}(110)$ planes perpendicular to the growth direction. This results in a $15 \%$ lattice mismatch at the interface.

The high-angle superlattice peak positions are determined by the relation:

$$
2 \frac{\sin \theta}{\lambda_{x}}=\frac{1}{d} \pm \frac{n}{\Lambda}
$$

where $\bar{d}$ is the average lattice spacing in the growth direction, $\Lambda$ is the modulation wavelength, and $\mathrm{n}$ is an integer that labels the order of the satellite about the average lattice peak. ${ }^{7}$ The only quantities that can be determined from the superlattice peak positions are $\bar{d}$ and $\Lambda$. The measured $\bar{d}$ values for a series of $\mathrm{Fe}(20 \AA) / \mathrm{Nb}(\mathrm{x})$ superlattices as a function of Fe percentage is shown in Fig $4 \mathrm{~b}$ as the open square. The $\overline{\mathrm{d}}$ values fall above the solid line 
traced assuming that the $\mathrm{Fe}$ and $\mathrm{Nb}$ layers have their bulk lattice spacing $\left(\mathrm{d}_{\mathrm{Fc}}=2 .() 27 \AA\right.$ and $\mathrm{d}_{\mathrm{Nb}}=2.334 \AA$ ) indicating $\mathrm{a} \approx 2 \%$ lattice expansion within the superlattice unit cell. This feature is common to a number of lattice-mismatched metallic superlattices. ${ }^{8}$

The structural parameters of the individuai layers were refined according to a welltested procedure. ${ }^{7}$ The refined spectrum, given by the solid line in Fig. 4a, reproduces the measured intensities over a range that spans three orders of magnitude. The fitted average lattice spacings of the $\mathrm{Fe}$ and $\mathrm{Nb}$ layers of $2.10 \pm 0.01 \AA$ and $2.34 \pm 0.01 \AA$, respectively, indicate that the majority of the lattice expansion is confined to the Fe layers. These values are shown as solid squares in Fig. 4b: the dashed line joining them agrees with the trend of the measured $\overline{\mathrm{d}}$ values for the entire $\mathrm{Fe} / \mathrm{Nb}$ series measured. The strain of the Fe layer, as determined by the refinement procedure, is not uniform across the layers, but is confined mostly to the first few monolayers of Fe grown onto the $\mathrm{Nb}$ layer.

The refinement procedure also determines the type and amount of structural disorder. The discrete disorder which results from layer thickness fluctuations of integer number of atomic planes is less than $2 \AA$, or 1 monolayer, for both layers. This indicates very smooth layering over the lateral crystalline coherence length. The main type of disorder which limits the crystal coherence length is continuous interface disorder. This is modeled in the refinement procedure as a Gaussian fluctuation of the interface lattice spacing with a fluctuation width of $0.3 \AA$. This value is equal to the difference in the Fe and $\mathrm{Nb}$ lattice spacings and is consistent with that obtained for other lattice-mismatched superlattices. ${ }^{7,9}$ This type of disorder is thought to result from a lattice mismatched interface that is incommensurate in-plane. ${ }^{9}$

To further explore the in-plane structure, selected $\mathrm{Fe} / \mathrm{Nb}$ superlattices were removed from the substrate and studied by transmission $x$-ray diffraction. The in-plane crystalline coherence length determined from the line width of the $[1 \overline{1} 0]$ reflection was $\approx 50 \AA$ for superlattices with $\Lambda<50 \AA$. The in-plane lattice strains for the Fe and Nb layer were $<2 \%$ and of opposite sign, compressive for $\mathrm{Nb}$ and expansive for $\mathrm{Fe}$. Although the sign of the 
in-plane strains are in agreement with the expectations of coherency strains, the small magnitude of the strain in comparison to the lattice mismatch (15\%) indicate that the interface are essentially incommensurate. This conclusion is in agreement with that obtained from the refinement results.

\section{DISCUSSION}

The structure and magnetic order determined by $\mathrm{x}$-ray-diffraction and polarized neutron reflectivity results give some insight into the magneto-transport properties, and, in particular, the lack of a truly "giant" MR. Both techniques show that the $\mathrm{Fe} / \mathrm{Nb}$ superlattices are well ordered perpendicular to the plane of the film with coherence lengths of many bilayer periods. However, the in-plane coherence (and degree of orientation) are significantly reduced. Therefore, it is likely that the limitation of the lateral crystalline coherence and the incommensurate interface is responsible for the large resistivities reported $(\rho \sim 100 \mu \Omega \mathrm{cm})$ for these films. ${ }^{1}$ Similar results have been found for other latticemismatched superlattices in which the electron mean free path is limited to the layer thicknesses by the interfaces. ${ }^{10}$ In addition to the $\mathrm{AF}$ alignment of the ferromagnetic layers, giant MR requires, among other factors, that the electron mean free path be greater than the bilayer thickness. In light of the high residual resistivity of these films, it is not unexpected that $[\rho(0)-\rho(H)]$ be small, even for films in which the AF coupling has been positively confirmed by neutron reflection measurements. Thus, the basic mechanism of the $\mathrm{Fe} / \mathrm{Nb}$ magnetoresistance may not be substantially different from that for $\mathrm{Fe} / \mathrm{Cr}$.

\section{ACKNOWLEDGMENTS}

This work was supported by the US-DOE, BES-MS under contract W-31-109ENG-38. One of us (J.E.M.) was supported by the NSF, MRG Contract no. DMR-8603304. 


\section{REFERENCES}

1. J. E. Mattson, C.H. Sowers, A. Berger and S.D. Bader, Phys. Rev. Lett. 68, 3252 (1992).

2. P. Grünberg, R. Schreiber, Y. Pang, M.B. Brodsky and C.H. Sowers, Phys. Rev. Lett. 57, 2442 (1986); M.N. Baibich, J.M. Broto, A. Fert, F. Nguyen Van

Dau, F. Petroff, P. Etienne, G. Creuzet, A. Friederich and A. Chazelas, Phys. Rev. Lett. 61, 2472 (1988); S.S.P. Parkin, N. More and K.P. Roche, Phys. Rev. Lett. 64, 2304 (1990).

3. G.P. Felcher, R.O. Hilleke, R.K. Crawford; J. Haumann, R. Kleb and G. Ostrowski, Rev. Sci. Instrum. 58, 609 (1987).

4. S.S.P. Parkin, A. Mansour and G.P. Felcher, Appl. Phys. Lett. 58, 1473 (1991).

5. J. Lekner, Theory of Reflection (M. Nijhoff Publ., Dordrecht, 1987).

6. Y.Y. Huang, G.P. Felcher and S.S.P. Parkin, J. Magn. Magn. Mater. 99, L31 (1991).

7. E. E. Fullerton, I. K. Schuller, H. Vanderstraeten, and Y. Bruynseraede, Phys. Rev. B 45, 9292 (1992).

8. I. K. Schuller and M. Grimsditch, J. Vac. Sci. Technol. B 4, 1444 (1986).

9. J.-P. Locquet, D. Neerinck, L. Stockman, Y. Bruynseraede, and I.K. Schuller, Phys. Rev. B 39, 3572 (1988).

10. T.R. Werner, I. Banerjee, C. M. Falco, and I.K. Schuller, Phys. Rev. B 26, 2224 (1982). 


\section{FIGURE CAPTIONS}

Fig. 1 Nb thickness dependencies of room temperature magnetic properties for an $\mathrm{Fe}(20 \AA) / \mathrm{Nb}\left(t_{\mathrm{Nb}} \AA\right)$ superlattice series: (a) squareness ratio $\left(\mathrm{MR}_{\mathrm{R}} / \mathrm{M}_{\mathrm{S}}\right)$ determined by SQUID magnetometry and (b) magnetoresistivity represented as $\Delta \rho / \rho$. The dashed lines in both panels serve as guides to the eye and the numbers label aas many as five AF oscillations.

Fig. 2 Neutron reflectivity of a superlattice $[\mathrm{Fe}(15 \AA) / \mathrm{Nb}(15 \AA)]_{100}$ at room temperature and in a 30 Oe magnetic field. The continuous line represents a model calculation in which the magnetic moments on subsequent Fe layers are opposite to each other, as described in the text. The Bragg peak due to the $\mathrm{AF}$ ordering is indicated by the arrow. $\mathrm{q}_{\mathrm{c}}$ is defined as the critical momentum for total external reflection.

Fig. 3 Intensity contour plot of the neutrons scattered by the $[\mathrm{Fe}(15 \AA) / \mathrm{Nb}(15 \AA)]_{100}$ superlattice for $\theta_{\mathrm{i}}=1.92^{\circ}$. The continuous line represents $|\mathrm{q}|=0.106 \AA^{-1}$ and corresponds to the antiferromagnetic Bragg reflection.

Fig. 4 a) $\mathrm{X}$-ray diffraction spectrum of a $\mathrm{Fe} / \mathrm{Nb}$ superlattice using $\mathrm{Cu}-\mathrm{K}_{\alpha}$ radiation. The indices give the order of the satellite reflections. The continuous line represents the results of structural refinement of the data. b) The average lattice spacing $\overline{\mathrm{d}}$ obtained from the central Bragg reflection for a series of $\mathrm{Fe}(20 \AA) / \mathrm{Nb}(\mathrm{x})$ superlattices (open squares). These values are compared with the values expected using the bulk $\mathrm{Fe}$ and $\mathrm{Nb}$ d-spacings (continuous line) and the refined lattice spacings from Fig. 4a (solid squares and dashed line). 


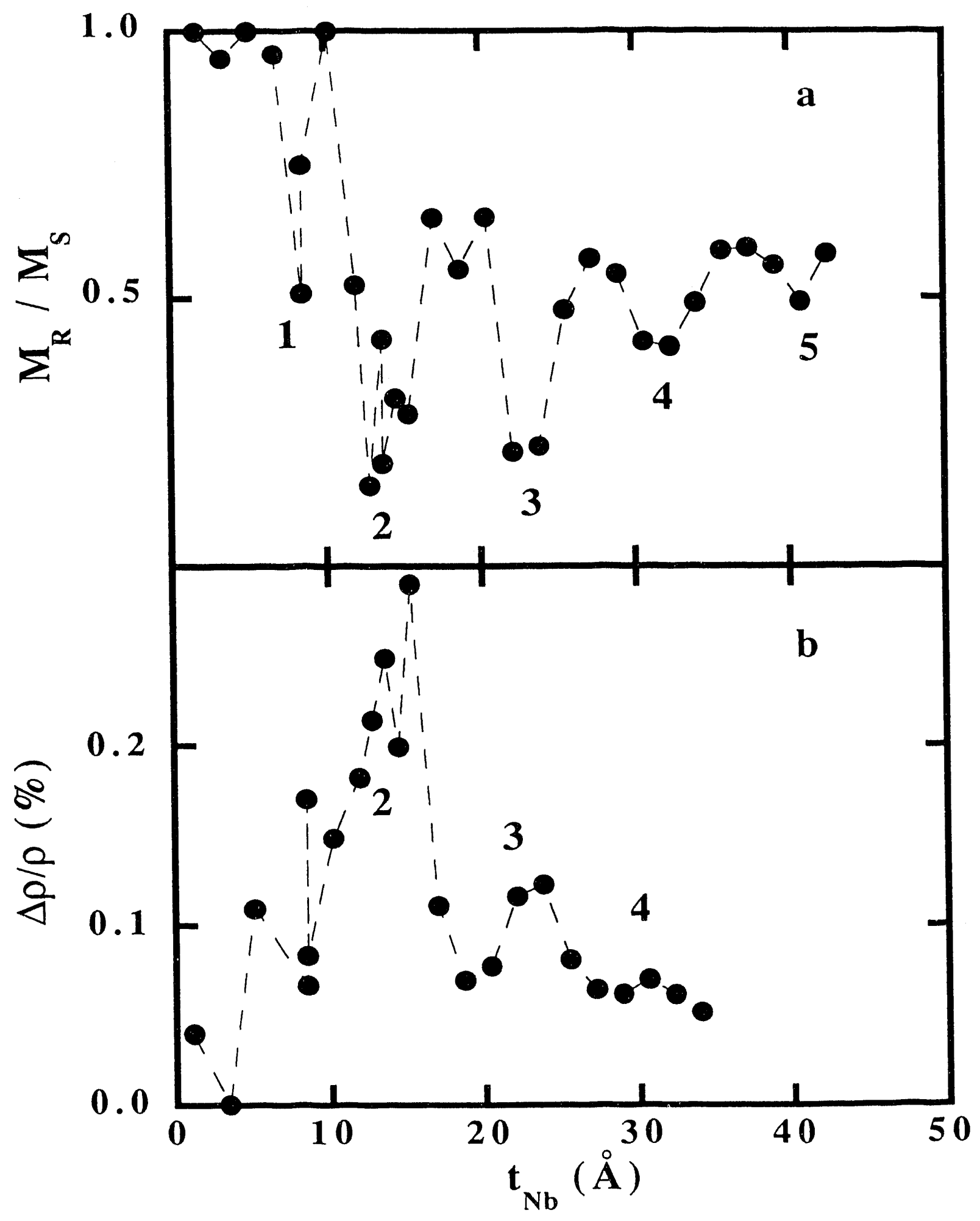




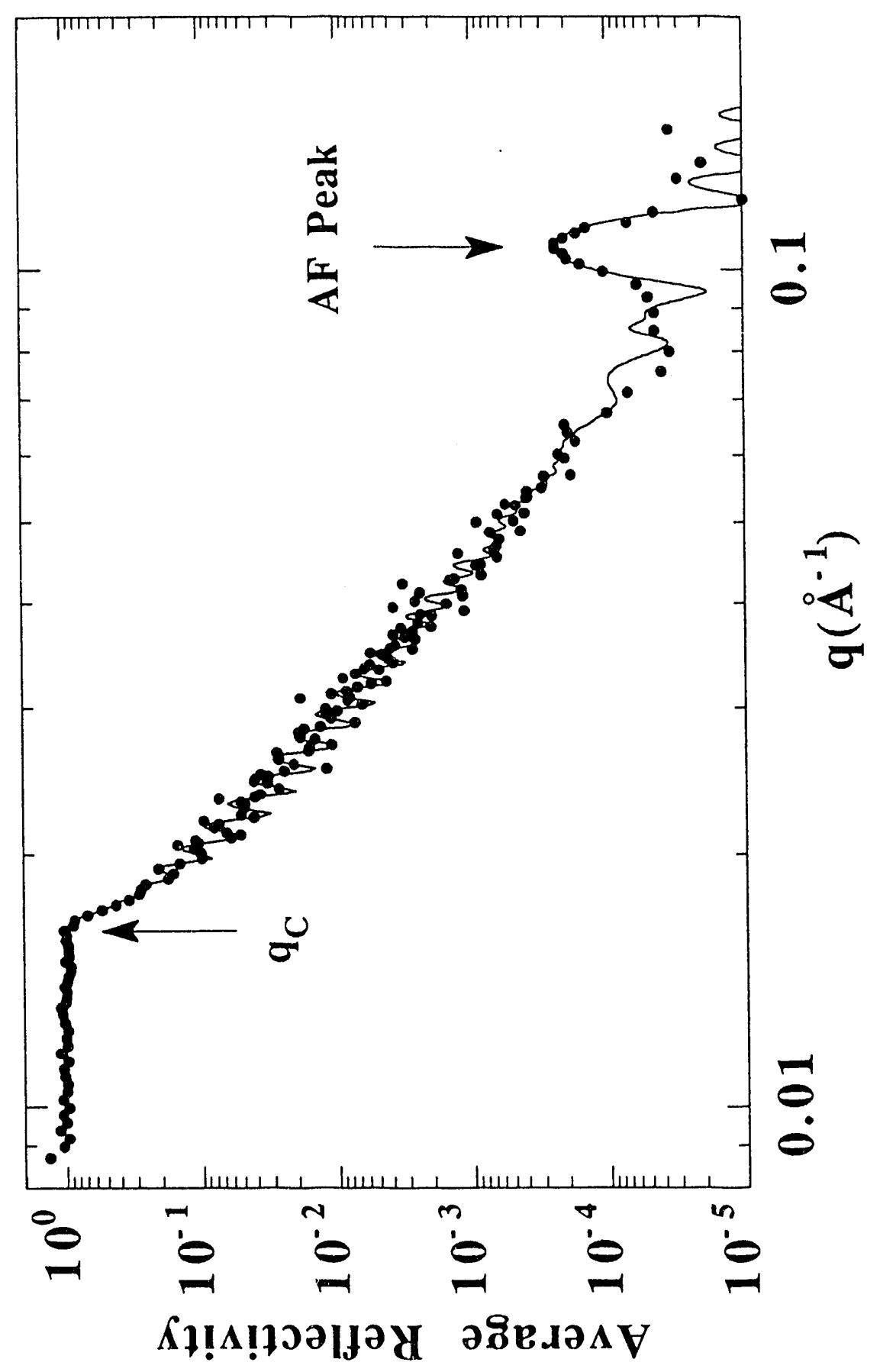




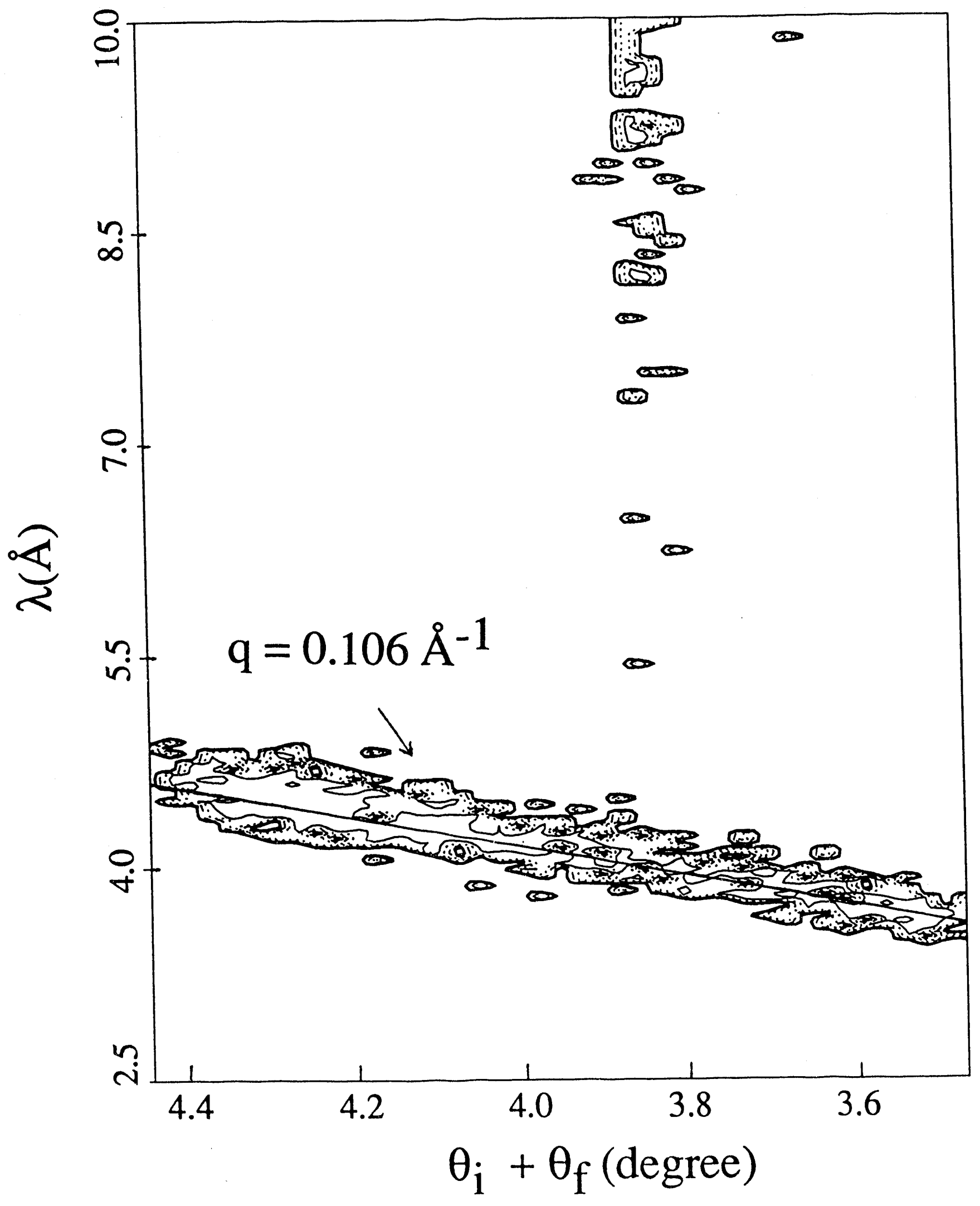




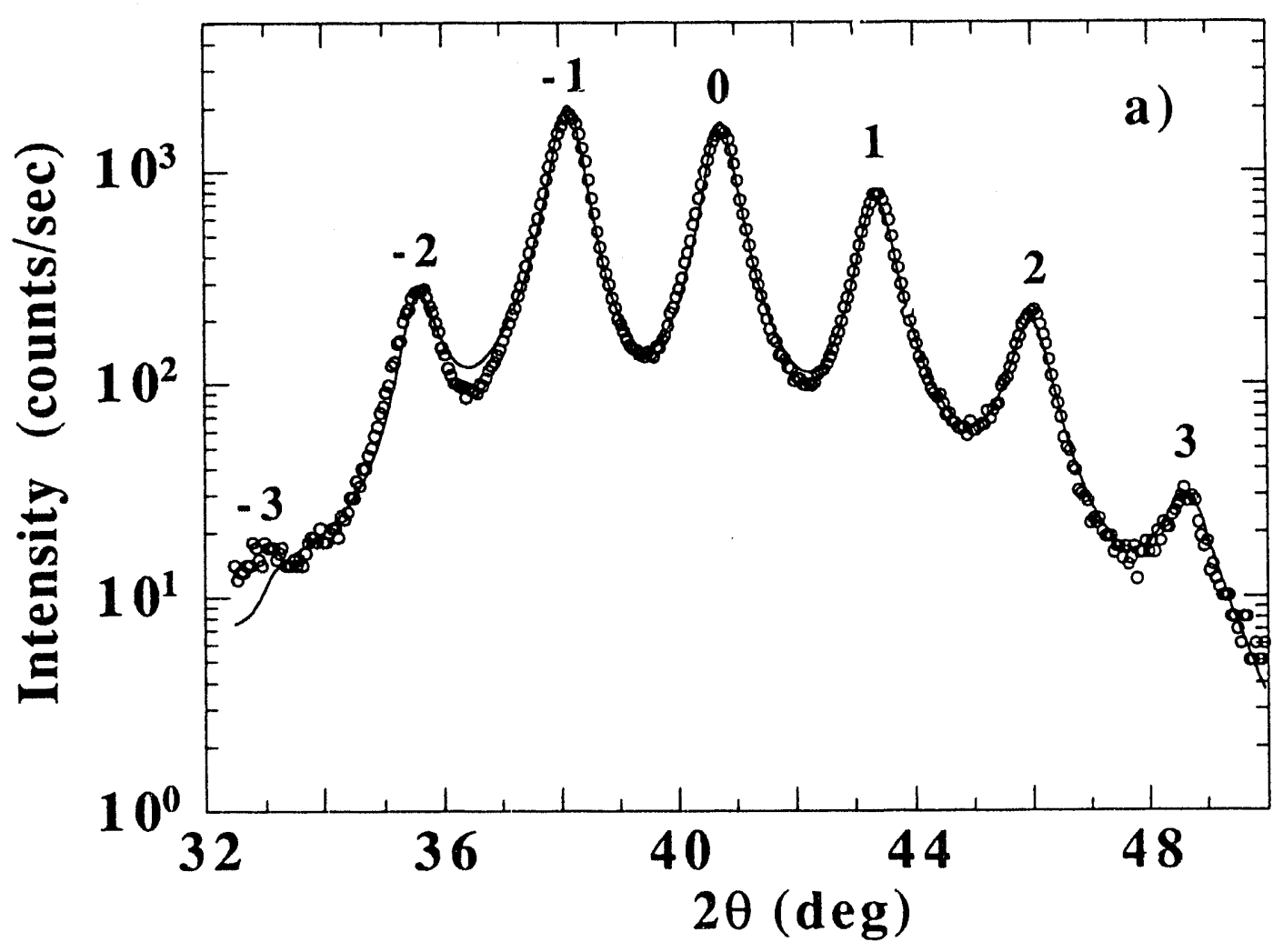




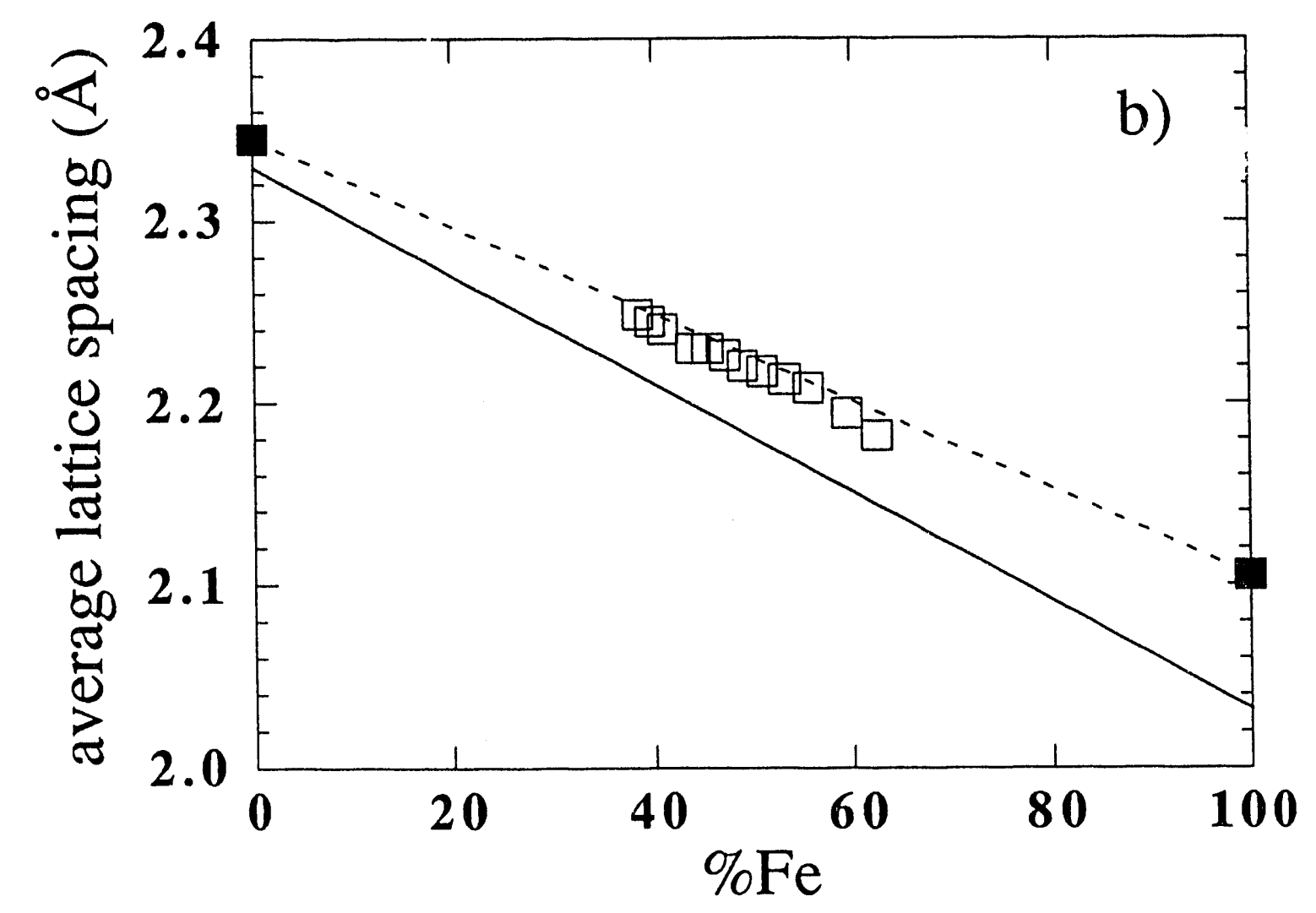



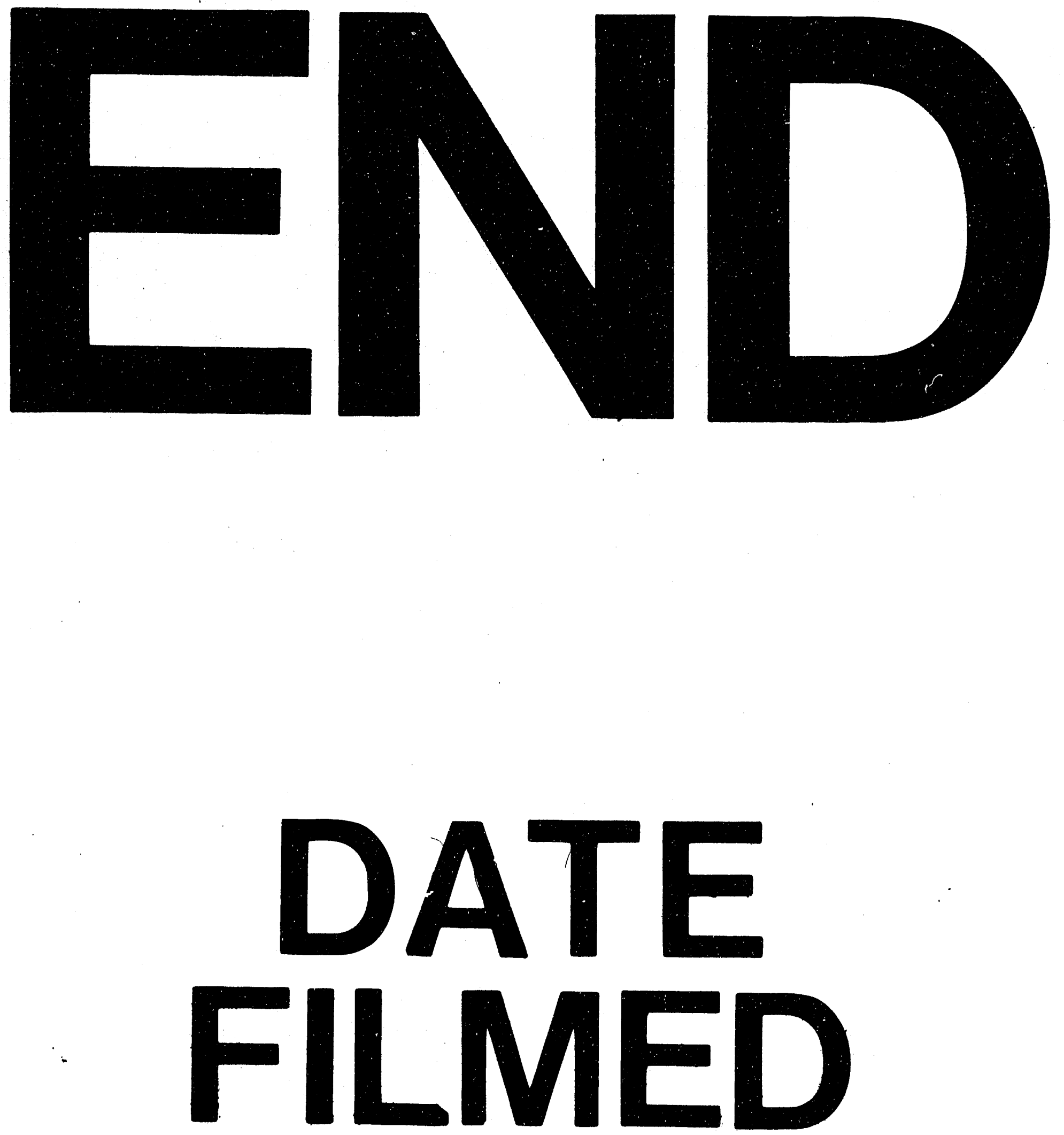

$\uparrow$
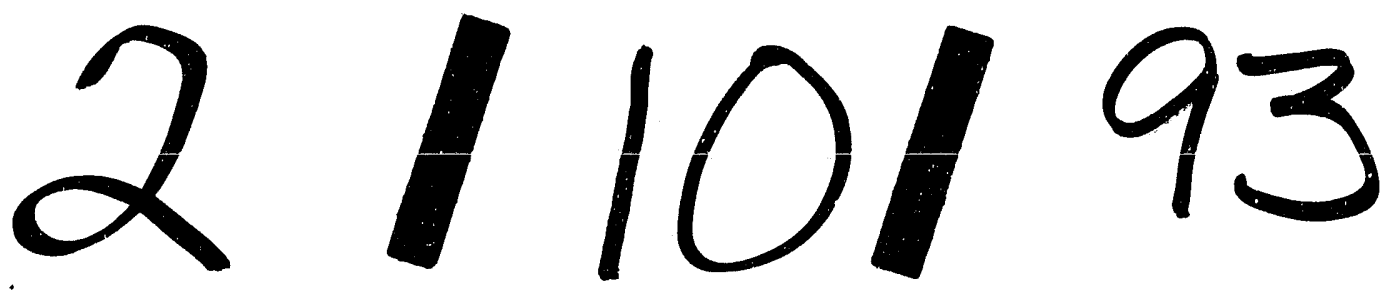
